U. S. DEPARTMENT OF COMMERCE

ROY D. CHAPIN, Secretary

BUREAU OF STANDARDS

LYMAN J. BRIGGS, Acting Director

BUREAU OF STANDARDS MISCELLANEOUS PUBLICATION No. 137

\title{
PAINT FOR PRIMING PLASTER SURFACES
}

By

PERCY H. WALKER

E. F. HICKSON

Issued August 31, 1932

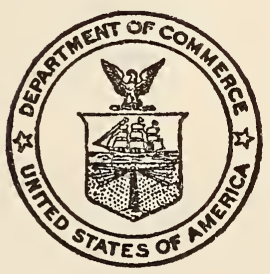

UNITED STATES

GOVERNMENT PRINTING OFFICE

WASHINGTON : 1932 


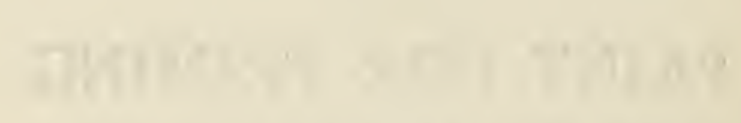

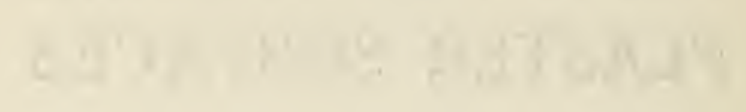




\title{
PAINT FOR PRIMING PLASTER SURFACES
}

\author{
By Percy H. Walker and E. F. Hickson
}

\section{ABSTRACT}

In response to demands from several branches of the Government service for information and specifications for priming paints for plaster surfaces, a number of commercial and experimental paints were tested on plaster, concrete, and other porous surfaces. Several novel methods of test are described and results obtained with 40 priming paints are recorded. It is found that a number of primers are commercially available which satisfactorily meet the requirements for a good material for this purpose with respect to hiding power, ability to so effectively seal a porous surface with one coat that the appearance of a second (finishing coat) of either flat or glossy oil or varnish paint will after drying have the same appearance as when applied to an impervious surface. It was found that the appearance or adherence of paint applied to any of the primers was materially affected by excessive moisture. A suggested specification based upon physical tests and comparison with a primer than can be readily mixed from specified materials is given in an appendix.

\section{CONTENTS}

I. Introduction

II. Materials used

III. Special methods of testing:

1. Humidity cabinet test Painting fresh concrete (Portland cement mortar) and plaster.

3. Color failure and blistering

4. Penetration.

5. Ability to prevent spotting

IV. Record of tests

V. Discussion

VI. Recommendations:

1. Condition of plaster

2. General characteristics of a good plaster primer

3. How to get a good plaster primer VII. Acknowledgment

VIII. Appendix - Suggested specification for paint for priming plaster surfaces (plaster primer and sealer)

\section{INTRODUCTION}

Although oil or varnish paints do not last as well on plaster as on interior wood or metal, ${ }^{1}$ they are extensively used on such materials. F. C. Atwood ${ }^{2}$ estimates that from 60 to 70 per cent of all paint produced is used for decoration only, the major portion of which is used on interior plaster.

Within the last year or two, special paints for plaster have appeared on the market which are designed to save material and labor

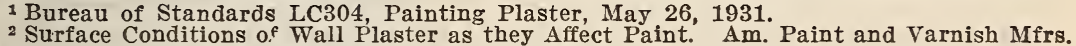
Assn. Circ. No. 404, pp. Jo1-521, December, 1931.
} 
by reducing the number of coats needed to produce a satisfactory appearance. Several branches of the Government service have requested specifications for such priming paints, and the work described in this paper was undertaken with that object in view.

Before this work was started it was learned that H. A. Gardner, of the American Paint and Varnish Manufacturers' Association, was investigating commercial plaster primers, and an informal agreement was made that the two laboratories would cooperate in the experimental work, but would show independent conclusions and issue independent publications. Gardner has published his results. ${ }^{8}$ The similarity in method of attack is evidence of the frequent conferences on the two investigations. However, the numbers given to the paints tested are not the same in the two reports.

\section{MATERIALS USED}

Thirty-three priming-coat materials were secured from manufacturers or industrial research laboratories. Some of these were furnished directly by the maker, and some were furnished by Gardner. All were taken from original containers. Most of these 33 paints are commercial products and represent fairly the types of priming materials for plaster that are now on the market. A few are evidently experimental and may or may not represent commercial material. These paints are designated by numbers in Table 1 and in the following discussion.

Seven paints which do not represent any particular brands, and therefore may properly be fully identified, are designated by the letters $K$ to $R$, inclusive. Following is a full description of each of these and the reasons for including each.

Paint $K$.-A factory-made raw linseed oil paint that has been used as a priming coat for plaster on certain Government buildings. The specification calls for a paint made from

80 pounds white lead paste.

20 pounds zinc oxide paste.

3 gallons raw linseed oil.

3 gallons turpentine.

1 pint drier.

This mixture yields about 9 gallons of paint. Laboratory examination indicated that it was well made and conformed to the specification.

Paint L.-Made in the laboratory by adding one-half gallon of boiled linseed oil to 1 gallon of flat lithopone paint, that, except for a slight deviation in composition of the pigment, conformed to Federal specification TT-P-41, Type II, Ready-Mixed Paint. The notes in Federal specification TT-P-41 advise that the ready-mixed paint as received "should be suitable for body or finish coat work. For priming coats on walls, from 1 quart to 1 gallon of boiled linseed oil should be added to each gallon of the ready-mixed paint." Similar directions are common on cans of commercial flat wall paints. This paint, therefore, represents practice that has been advised for many years.

8 H. A. Gardner, L. P. Hart, and J. R. Stewart, Primers for Freshly Plastered Surfaces. Am. Paint and Varnish Mfrs. Assn., Circ. No. 405, February, 1932. 


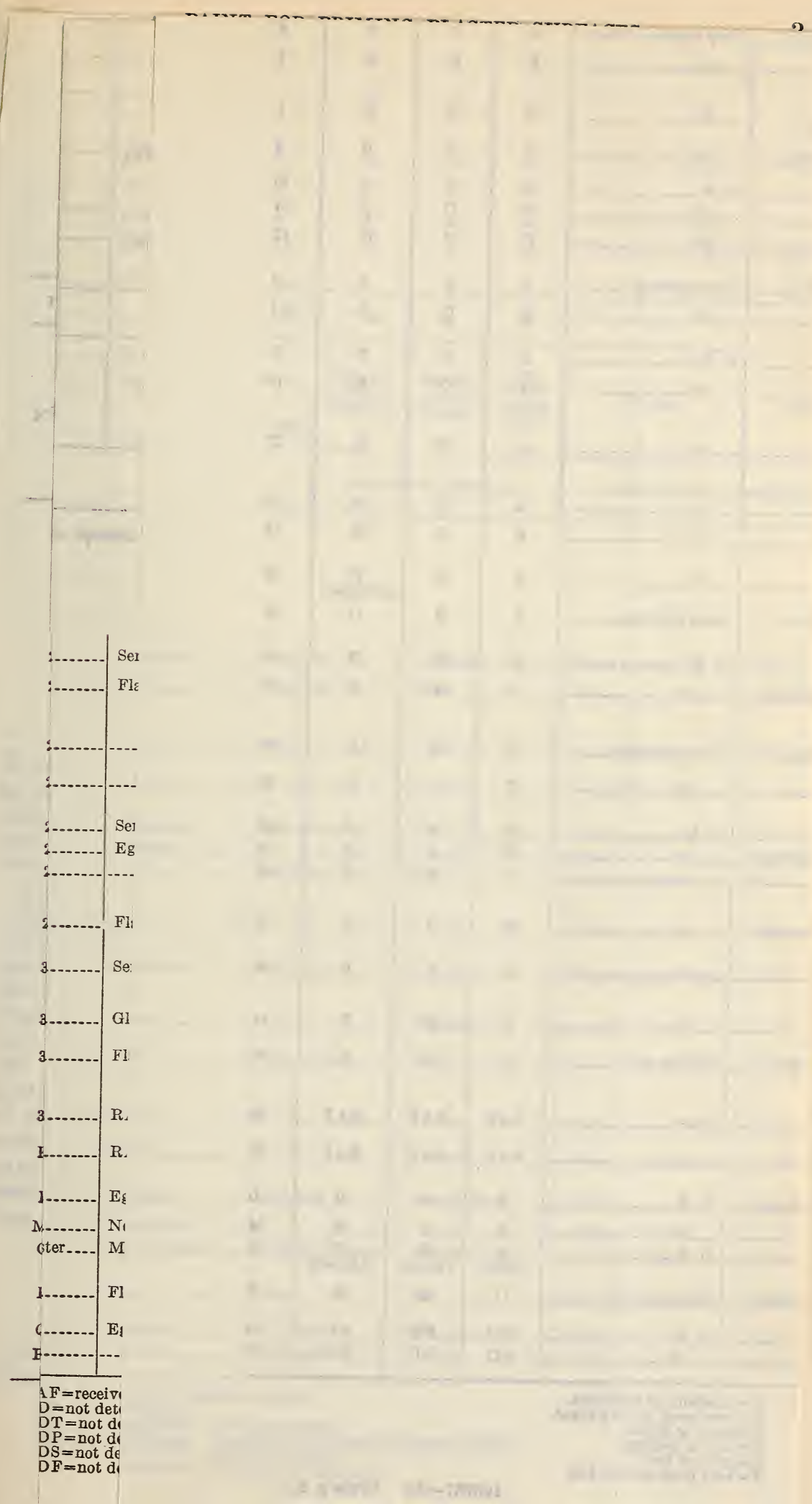



TABLE 1. - Comparative tests on primers for plaster surfaces

\begin{tabular}{|c|c|c|c|c|c|c|c|c|c|c|c|c|c|c|c|c|c|c|c|c|c|c|c|}
\hline \multirow{3}{*}{$\mathrm{N}_{\mathrm{a}}$} & \multicolumn{4}{|c|}{ Composition } & \multirow{3}{*}{ 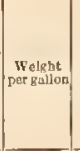 } & \multirow{3}{*}{ Brushlag gropertles } & \multicolumn{2}{|c|}{ 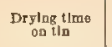 } & \multirow{3}{*}{ 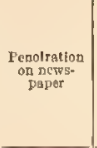 } & \multirow{3}{*}{ 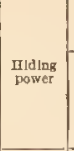 } & \multicolumn{2}{|c|}{ Tasts for brillenoss a } & \multicolumn{4}{|c|}{ Glass (ono eant) } & \multicolumn{3}{|c|}{ 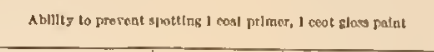 } & \multicolumn{3}{|c|}{ Vemtreatdel In wet sand } & \multirow{3}{*}{$\begin{array}{lll}\text { tean } & \text { No. } \\
\text { ing } & \end{array}$} \\
\hline & \multicolumn{3}{|c|}{ Palat } & \multirow{2}{*}{ Major plgnent consstisuents (It per cent) } & & & \multirow[b]{2}{*}{ sot } & \multirow[b]{2}{*}{ Dry } & & & \multirow{2}{*}{ 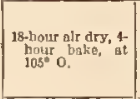 } & 12hour olr dry, t- & & & & & & & & & & & \\
\hline & Plgment & Volatslo & $\begin{array}{c}\text { Nonvola. } \\
\text { thilo } \\
\end{array}$ & & & & & & & & & 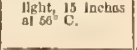 & On thn & On concreto & On plaster & On nerrspaper & Blottlng pajex & Concresto & Flaster & 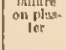 & 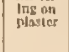 & $\begin{array}{l}\text { for ro: } \\
\text { rantublum }\end{array}$ & \\
\hline 1 & 2 & 3 & ' & 8 & 6 & $\stackrel{7}{2}$ & 8 & ${ }^{9}$ & 10. & 11 & 12 & 13 & $"$ & 15 & 16 & 17 & เ\$ & 19 & 20 & 21 & 2 & 23 & 24 \\
\hline 1 & $\begin{aligned} & \text { Per cent } \\
& 41\end{aligned}$ & $\begin{aligned} & P R \operatorname{Pen} t \\
& 22\end{aligned} \mid$ & {$\left[\begin{array}{rr}\text { Perent } \\
3 i\end{array} \mid\right.$} & 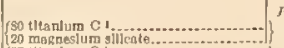 & $\begin{array}{c}\text { Pounds } \\
10.8\end{array}$ & onol... & $\begin{array}{c}H \text { Hours } \\
22 / 3\end{array}$ & $\begin{aligned} & \text { Hours } \\
& 7\end{aligned}$ & Complete. & $\left|\begin{array}{l}\text { sq. p.p.pont. } \\
1+0\end{array}\right|$ & o. $\mathrm{k}$ & . $0 . \mathrm{K}$ & Scm|sloss. & Flat...... & E Egsholl. & Fitat.... & $0 . \kappa \ldots$ & silght spotthe.. & $0, \mathbf{K}_{\ldots}$ & $\mathbf{n}$ & $\mathrm{D}$ & $\mathbf{n}$ & \\
\hline 2 & 40 & 2 & 30 & 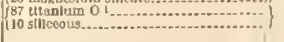 & 11.0 & (-..do...... & 2 & 7 & Novo......... & 170 & -....de.... & -...do... & Glase. & fotess $\ldots . . . .$. & atass....... & Otoss..... & ............ & $0.5 \ldots \ldots . . . . . .$. & $\ldots$...do & D & $n$ & D & \\
\hline 3 & 28 & ${ }^{20}$ & 23 & 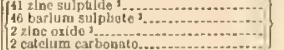 & 0.4. & ......do... & 2 & & ..... do... & 120 & do & .40. & ....do- & Eggshell... & Somighose. & - Eetollasos... & do. & . Alo... & & 0 & o & B & \\
\hline 4 & 42 & 28 & 30 & 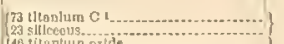 & 10.6 & ............... & 1 & ${ }^{\circ}$ & Slight... & ${ }_{120}$ & ....do..... & ....do...... & Semlgloss.. & .....do... & .....do... & Ergstlell. & -...do & , n...do. & $\ldots . . . . \mathrm{d}$ & $A$ & $\mathrm{n}$ & n & \\
\hline$\cdot$ & 22 & ${ }^{30}$ & 12 & 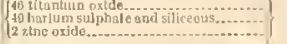 & 0.1 . & .....do.... & 1 & 5 & sorne & 135 & $\ldots$ do. & ........ & ...do. & ................. & Eggshell.. & Flut... & $\ldots . . . \mathrm{do}$ & .......to. & ..... & n & в & v & \\
\hline 6 & s1 & ${ }^{21}$ & & 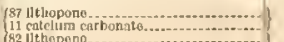 & 12.5 & Very good ....... & 3 & 18 & Comploto. & 2000 & $\ldots$......... & ........... & . do...... & - n do & 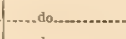 & .............. & ......do..... & sllgut glotlan.. & Vory NIght spotting. . & D & 0 & $n$ & \\
\hline 7 & 51 & ${ }^{22}$ & & 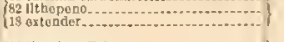 & 12,0 & ood & 1 & $s$ & Slight....... & $1+3$ & ....do... & -.............. & Egesbell.. & 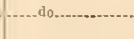 & - do do & .................. & ....... do..... & ......do.............. & 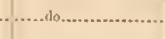 & n & - & 0 & \\
\hline 8 & ${ }_{52}$ & 29 & 19 & 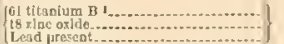 & 12.1 & .....do.... & 1 & $s$ & Nona...... & 172 & ....do.... & ....do. & Semistass- & .....do... & .....do... & .......do.. & ....do. & ....do. & & e & 0 & $n$ & \\
\hline$\circ$ & 18. & 42 & 10 & 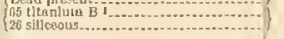 & 11.4 & Excollent_..... & 1 & 8 & - & 122 & $\ldots$ & ......do... & Fist.......... & Flat......... & Flat.......... & 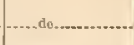 & Flat t............ & Sono stotllng... & $\ldots . . . \mathrm{do} \ldots . .$. & n & $\mathrm{n}$ & $"$ & \\
\hline $\begin{array}{l}10 \\
11\end{array}$ & $\begin{array}{l}28 \\
37\end{array}$ & $\begin{array}{c}14 \\
34\end{array}$ & ${ }_{z 3}^{28}$ & 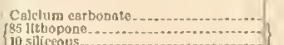 & $\begin{array}{r}8.7 \\
10.2\end{array}$ & 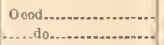 & 1 & $\frac{8}{8}$ & $\begin{array}{l}\text { Sllgb1........ } \\
\text { Nour_...... }\end{array}$ & ${ }_{105}^{\text {NDT. }}$ & 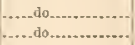 & ....do... & \begin{tabular}{|l} 
Gloss......... \\
Scmiglos...
\end{tabular} & Sounisloss ... & sumiglos .... & Semigloses. & o. $\mathrm{k} \ldots \ldots \ldots \ldots$ & $0, \mathrm{~K} \ldots \ldots \ldots$ & o. $\mathrm{K} \ldots$ & n & 13 & $"$ & \\
\hline 12 & 12 & 28 & & 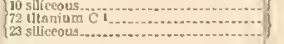 & 11.0 & Falt (pulls) ..... & 1 & 5 . & …do......- & 170 & …...do..... & ............. & Eggsholl........ & Egghhell.... & Egganthel.... & 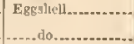 & Serno spottlus.... & 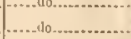 & ......th & $\begin{array}{l}0 \\
0\end{array}$ & D & $"$ & \\
\hline 13 & ${ }_{18}$ & а1 & 21 & 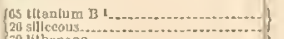 & 11.5 & Falr (quick set & 1 & 8. & .....de... & 122 & do.... & ....do... & Hilat . & Flnd ..... & Fint.... & Flat.... & Some spotting... & Bonto spottung..... & .. santo strotung & $\Lambda$ & $n$ & $"$ & \\
\hline 14 & 69 & 24 & 18 & 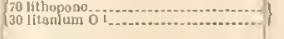 & 33.5 & Fuir (tadse bo & 1 & 5 & sorwe........ & NDP. & Flne cracks... & Fino cmeks. & -...do-_ & ................ & $\ldots . .$. do.... & $\ldots$............ & Eud sputthys.... & $\ldots . .$. & ........4. & 11 & 0 & " & \\
\hline 15 & 43 & 27 & 30 & 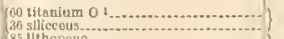 & 11.0 & Oood......... & 1 & i & Nono...... & 137 & o. K...... & - Minulo creas & Egrshell.-. & Eggsbell_. & Egssthell_- & Eggashutit- & 0. $\mathrm{K}$. & $o, k \ldots$ & o. $k \ldots$ & $\Lambda$ & 0 & $n$ & \\
\hline 18 & 12 & 28 & & 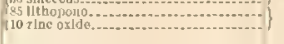 & 11.2 & Falr (pullis) ... & 1 & . & ......do.... & NDP. & nad eracks... & Bad erecks... & Gloss........ & Olass _... & Oloss_. & aloss... & s1lght spolting...... & .....do... & ......10... & A & 0 & n & \\
\hline 17 & ${ }_{45}$ & 28 & ${ }^{27}$ & 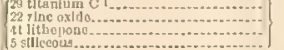 & 11.5 & aood... & 1 & 5 & Scomo. & 112 & O. E.... & o. . r... & Eggshell. & Eggsshell- & Egshell.. & Eggshell. & o. $\mathrm{k}$ & & 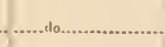 & $\wedge$ & 0 & $"$ & \\
\hline 18 & is & 28 & ${ }_{24}$ & 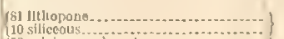 & 11.5 & .....do..... & 1 & 5 & $\ldots . . . \mathrm{do} 0 . .$. & 133 & ....do... & ............ & do.. & ....... & ................ & Fitht & & & & $\wedge$ & o & $\mathrm{n}$ & \\
\hline 10 & 25 & 47 & 20 & 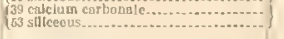 & 8.4 & $(-. .40 . \ldots \ldots$ & 1 & 5 & sllgbt......... & NDT. & do & Aflinuts erat & Sotrigloss_. & ................. & Sentgloss. & Eggeshell_. & (......do......... & $\ldots$ & ......do....... & n & 0 & $"$ & 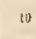 \\
\hline$x$ & 4 & 28 & 30 & 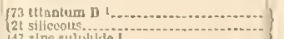 & 12.7 & Poor (pulls)... & 1 & 5 & Nono........ & NDP. & ....do.... & $0 . \mathrm{K}_{\ldots}$ & ...do. & .....10 & ...... do & Fint... & Voty slight sinteteng. & ............ & antio. & B & n & . & \\
\hline at & B & 30 & 10 & 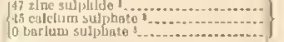 & 12.0 & Very good... & 3 & 2 & Slight_..... & 182 & ....do. & do- & Fint & Fint. & Flot. & $\ldots$ & 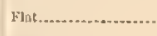 & Dnd Epottng....... & Sorrue siattlug.... & $\wedge$ & a & ? & \\
\hline$n$ & ${ }_{10}$ & 20 & 81 & no titanlum C C.... & 10.2 & .....do.... & 1 & 5 & Complete. & 165 & .......... & ........... & eloss.......... & Somisloss. & Sonilgtous... & Eggsholl... & stght spottlos.... & $\mathrm{o}, \mathrm{K}$ & o. $\mathrm{K} \ldots \ldots \ldots$ & в & D & $"$ & \\
\hline 2 & 14 & 17 & 39 & 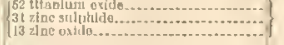 & 12.6 & Good.....- & $3: 2$ & 2 & stigbt... & 234 & ......... & - do... & Etgsholl. & Fina & Eggstell ... & Flet. & o. $\mathrm{k} \ldots \ldots$ & Silleit apouttiny... & $\ldots \ldots$ do.... & A & $\wedge$ & " & \\
\hline${ }^{24}$ & 71 & 15 & $1+$ & 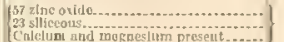 & 6.4 & Folr (thick & . & 2. & ..._do & NDS. & Flas erack & Ftho crien & Flat... & $\ldots$...de- & Flet. & ......do & Dad spoteting... & had spottlug. & Eann s|at ling. & $\mathrm{n}$ & " & $\mathrm{n}$ & \\
\hline${ }^{26}$ & ${ }^{70}$ & $1^{2}$ & ${ }_{12}$ & 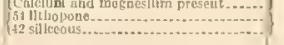 & 17. 0 & $\ldots \ldots$ do.... & & 2 & Sorno.......... & 195 & ......do....... & ..................... & -...do... & ........... & $\ldots . . .10$. & .....do.... & Neanry flat... & .....do............. & ....... da............ & 0 & o & $n$ & 25 \\
\hline 20 & 23 & 17 & 30 & 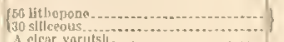 & 8.0 & oood. & 1 & & None...... & NDS, & o. K.... & $0 . \mathrm{K}$ & Senitisto: & Semi & Somisloss.......... & Ergesholl- & o. $\mathrm{K}$ & & o. $\mathrm{k}$ & $A$ & n & 0 & \\
\hline${ }_{28}^{27}$ & if & $\begin{array}{l}88 \\
60\end{array}$ & & 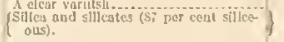 & $\begin{array}{l}7.2 \\
7.0\end{array}$ & 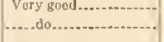 & $\stackrel{2}{1}$ & s. & \begin{tabular}{|l|} 
Completo... \\
Somo.......
\end{tabular} & $\begin{array}{l}\text { NDT. } \\
\text { NDT. }\end{array}$ & ....do...... & .... & 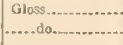 & Eggstell. & 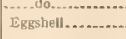 & 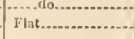 & 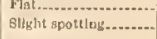 & $\begin{array}{l}\text { sorru spottlog... } \\
\text { o. K.................. }\end{array}$ & 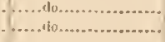 & is & $\hat{A}$ & 吾 & \\
\hline 20 & ai & 15 & ${ }_{21}$ & 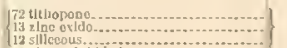 & 10.1 & $\begin{array}{c}0.00 \mathrm{w} \\
\text { wilue }\end{array}$ & 1 & 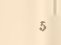 & Nono. & NDP. & do.. & do & Egsshul. & Flat. & ....don & $\ldots . . .40$ & $0, \mathrm{~K}_{\ldots .}$ & 8llkы ppottug & ..... & B & 0 & II & \\
\hline 30 & $4^{2}$ & 25 & 33 & 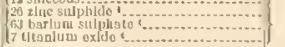 & 10.8 & Good (silght pulll) & $=$ & 7. & ......do... & 164 & $\ldots \mathrm{do}$ & ...do. & Gloss .... & Semiglass- & Somigloss... & Sarmiltoss. & Vory ollight tooltthe.... & o. K....... & .....do... & 。 & E & 11 & \\
\hline st & 33 & 32 & as & 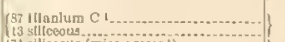 & 0.0 & Oood...... & $21>0$ & & sllgt.... & NDF. & ddo. & $\cdots$ & $\mid . .$. do.... & Oloss & $01058 . .$. & Egestell_. & ......do... & ....................... & ...do.... & $n$ & $\pi$ & D & $\boldsymbol{M}$ \\
\hline 32 & 28 & 12 & 30 & 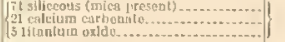 & 9.2 & ......10.......... & 2 & & …do... & NDT. & $\ldots$........ & ....do. & Nearly nat. & Flat.... & Nearly Rat..... & Flat........ & Flal,....... & Bligtuly dull.... & 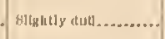 & o & c & B & 3 \\
\hline$s$ & 301 & a8? & 24 & 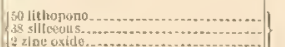 & 10.2 & ....to... & ${ }^{*}$ & & Complet & 123 & -..do. & .......so. & \begin{tabular}{|l|} 
ERgshdll. \\
\end{tabular} & R.M & RAF. & . & & ItA & RAF. & $\mathrm{n} \Delta \mathrm{F}$ & $\mathrm{n} A \mathrm{P}$ & $\Pi \wedge P$ & \\
\hline $\mathrm{k}$ & 68 & 17 & 23 & 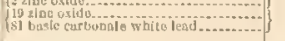 & 10.2 & Yery good... & 5 & 18 . & ......do.... & 155 & do.... & .do & Somigloss... & $\mathrm{BAF}$ & $R A F$ & ...do. & Dull.. & ....do. & ....do.. & แล & nAy & RAr & $k$ \\
\hline 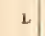 & s4 & 15 & st & 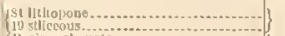 & 12.8 & Good... & 3 & ${ }_{18}$ & sot & 163 & .....do... & $\ldots$ & & Eggsho & Egsth & Near & O. $\mathrm{K}$ & Eamest & $0 . \mathbf{K} \ldots$ & n & ^ & n & \\
\hline M & $\begin{array}{c}67 \\
8\end{array}$ & 4 & $\begin{array}{c}39 \\
33\end{array}$ & 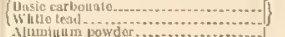 & $\begin{array}{c}15.2 .2 \\
8.8\end{array}$ & $\begin{array}{l}\text { Very good..... } \\
\text { Oood...... }\end{array}$ & $\begin{array}{l}3 \\
3\end{array}$ & 189 & Complato... & 11t+ & & & & Mator & & & & & . do... & n & $\wedge$ & n & M \\
\hline , & ( & | & & Mlarminum powdor... & 8.8 & Oood ..... & s & 3 & Some_-. & NDP. & $\cdots \cdot d 0$ & ....do. & Molusilio lesster... & Motallec Iusted & Mitallilo flut tor. & Metolllo lus or & sught spotting. & $0, K \ldots$ & O. K....... & $(0, x+1)$ & (wort) & (1) (1) & \\
\hline 4 & ${ }^{\infty}$ & . & (3) & 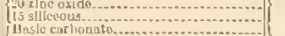 & 15.5 & into.... & & ${ }^{2}-$ & -..10 & NDP. & Flno crablss... & NDF.... & | Рав..... & Mlat..... & Flat. & Flat....... & Fiat... & Bor an soutllng..... & 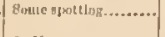 & D & B & B & the \\
\hline $\mathrm{R}$ & 30 & 27 & 3 & 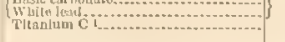 & $\begin{array}{l}7.9 \\
10.5\end{array}$ & ㄱety. & $\begin{array}{l}3 \\
3 \\
3\end{array}$ & $?$ & \begin{tabular}{|l|} 
Complate... \\
Noulo_......
\end{tabular} & $\begin{array}{l}145 \\
1 \leq 0\end{array}$ & o. . . . & 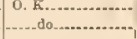 & Senitgloss.. & Ergsbcl... & $\begin{array}{l}\text { Esgatell... } \\
\text { samtgloss.. }\end{array}$ & Eesmigloss & O. K....... & 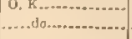 & 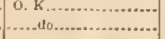 & $\begin{array}{l}\text { ND } \\
\text { ND }\end{array}$ & $\begin{array}{l}\text { ND } \\
\text { ND }\end{array}$ & $\begin{array}{l}\text { ND } \\
\text { ND }\end{array}$ & a \\
\hline
\end{tabular}




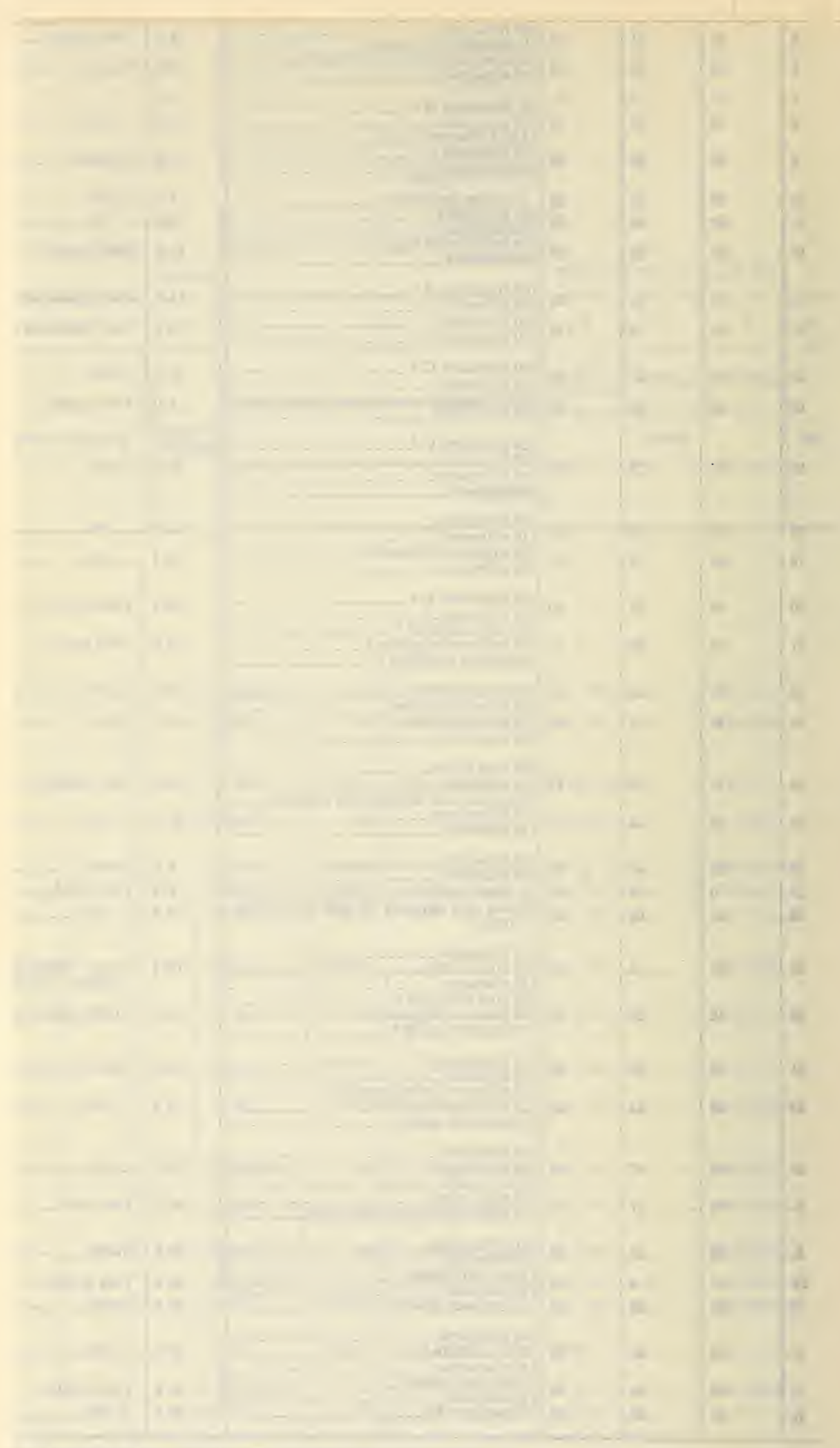

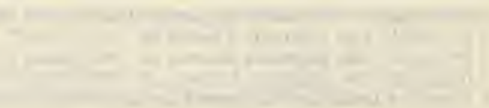


Paint M.-Made in the laboratory from basic carbonate white lead (heavy paste), boiled linseed oil, and turpentine in the proportion

100 pounds basic carbonate white lead (heavy paste).

7 gallons kettle-boiled linseed oil.

1 gallon turpentine.

This mixture yields about $102 / 3$ gallons of paint. This is also an old formula for priming coat for plaster. It was given in B. S. Technologic Paper No. 274, which was published in 1924, and probably was an old formula at that time.

Paint 0 .- Made in the laboratory by mixing 2 pounds of aluminum powder for paints (A. S. T. M. specification D-266-31) with 2 gallons of a synthetic resin varnish for outdoor use. Since it had been claimed that priming coats for plaster should be extremely waterproof, this paint, which was known to be remarkably waterproof, was selected to determine whether extreme resistance to water is desirable.

Paint $P$.-This sample was purchased for use as a white trafficmarking paint on highway surfaces. It was arbitrarily selected as a paint which contained pigments commonly used in commercial plaster primers, but which was designed for a radically different use, and was probably unsuitable for use as a plaster primer.

Paint $Q$.- Paint mixed in the laboratory from the following materials in the proportions indicated:

100 pounds stiff paste basic carbonate white lead in oil (F. S. TT-W-251, type B).

4 gallons interior varnish (F. S. TT-V-71).

2 gallons kettle-boiled linseed oil (F. S. JJJ-O-331, type A).

1 gallon turpentine (F. S. LLL-T-791).

This mixture yields about $92 / 3$ gallons of paint. This was selected as an easily described paint that could readily be made from materials for which there are definite specifications. Previous experience had shown this material to be excellent for use as a priming coat on unpainted plaster.

Paint R.-Paint made in the laboratory by grinding 39 parts by weight titanox $C$ with 34 parts of very heavy bodied linseed oil, 12 parts of mineral spirits, 12 parts of turpentine, and 3 parts liquid paint drier. The heavy bodied linseed oil was a light-colored commercial product having a "viscosity" at $25^{\circ} \mathrm{C}$. of 786 to 930 poises, depending on the pressure used (showing that the oil has plastic flow), a specific gravity of 0.97 and an iodine number (Wijs) of 130. The mineral spirits and turpentine conformed to Federal specifications TT-T-291 and LLI-T-791. The drier conformed to Type I of Federal specification TT-D-651. This formula was used in an attempt to make a nonpenetrating type of primer that would compare favorably with commercial nonpenetrating primers. Its composition is given simply for information without any recommendation as to its practical merits for commercial production.

\section{SPECIAL METHODS OF TESTING}

\section{HUMIDITY CABINET TEST}

A test, advocated by a well-known manufacturer of plaster primers as valuable for showing their properties with respect to blistering 
and retention of color, was given a thorough trial with several priming-coat materials. A cypress frame 29 by 29 by 18 inches with solid top and bottom was constructed. The four walls of the box were made of freshly prepared plaster panels painted (within 48 hours of plastering) on the face with various priming paints. The second or finishing coat of paint on all panels was an oil-gloss white lead-linseed oil paint tinted to a medium blue with Prussian blue in oil.

Inside this box an electric-light bulb, suspended in a shallow dish of water, produced practical saturation of the air and caused water to condense on the reverse side of the plaster panels. The outside air was kept dry and warm by placing 100-watt electric lamps about 6 inches from each panel.

This condition was maintained for 34 days, during which time no marked defects were noted on any of the panels.

The humidity apparatus was then removed from the interior of the box, and with the exterior lamps turned on, the test was continued 14 days longer with no appreciable change.

Since this test did not show any differences between the primers tested, it was not used on all the materials and the results do not appear in the table.

\section{PAINTING FRESH CONCRETE (PORTLAND CEMENT MORTAR) AND PLASTER}

Panels 10 inches square and 1 inch thick were prepared from 1 part Portland cement and 3 parts sand, using water in which onehalf gram of sodium sulphate was dissolved in each $1,000 \mathrm{ml}$. (The sodium sulphate was added to insure an alkaline reaction to water passing through the panel.) The panels were covered with wet paper, and occasionally watered for 48 hours. The paper was then removed and immediately one-half of each panel was coated with white finish-coat plaster consisting of 2 parts of hydrated lime and 1 part of plaster of Paris. After 48 hours one coat of the primer was applied, allowed to dry 24 hours, inspected (see columns 15 and 16 of Table 1), and then given one coat of a white lead-raw linseed oil paint tinted with Prussian blue, which would dry to an oil gloss on tin in less than 12 hours. After drying for 24 hours the panels were inspected for "flatting" or spotting (see columns 19 and 20 of Table 1). Duplicate tests made at different times agreed.

\section{COLOR FAILURE AND BLISTERING}

Each of the above panels was then embedded, with the painted surface uppermost, to half its thickness in wet sand contained in a shallow pan, and after 24 hours examined for color failure (which was accompanied by loss of gloss) and blistering. The panels were allowed to stay in the wet sand for six days longer, during which time the changes simply became more pronounced. The panels were dried for four weeks after they were removed from the wet sand. The color failures on plaster are shown in column 21. They are averages of duplicate tests. Color failures on concrete were more severe than on plaster, but are not recorded. The blistering on 
plaster is shown in column 22. Each is an average of two tests, and represents a 24-hour exposure.

\section{PENETRATION}

The test to determine whether a primer was a penetrating or nonpenetrating type was made by brushing a normal coat on newsprint. After drying for one hour the reverse side of the paper was visually examined for evidence of penetration or staining (see column 10 of Table 1). Similar tests on blotting paper and kraft paper were also made but are not recorded. Newspaper appeared to be the best of the three kinds of papers tested.

\section{ABILITY TO PREVENT SPOTTING}

In addition to the tests on concrete and plaster described in section 2 , sheets of blotting paper and kraft paper were given one brush coat of the various primers, allowed to dry for 24 hours, and given a brush coat of white lead-raw linseed oil paint, that would dry on tin in less than 12 hours to an oil gloss. The second coat was allowed to dry for 24 hours, and then examined for spotting or loss of gloss. See column 18 for results on blotting paper.

\section{RECORD OF TESTS}

In Table 1, columns 1 and 24 give the designation of each paint tested by number or letter. Columns 2 to 6 , inclusive, give results of analysis and weight per gallon. Column 7 records the operator's opinion as to brushing properties. Columns 8 and 9 record drying time on tin. Column 10 records penetration tests. (See III, 4.) Column 11 records hiding-power tests by the brush-out method (see $\mathrm{D}, 2, f$ of appendix). Columns 12 and 13 record brittleness tests on tin. Columns 14, 15, 16, and 17 record appearance of single coats of the primer on impermeable and permeable surfaces. Columns 18, 19, and 20 record ability to prevent spotting. Column 21 records color failure on wet plaster. Column 22 records blistering on wt plaster. Column 23 gives estimates as to condition for repainting after wetting for one week and then air drying for four weeks. A record (not shown) was also kept of the keeping qualities of the various paints after repeated use. With few exceptions the paints kept very well.

\section{DISCUSSION}

The results given in columns 21 to 23 , inclusive, of Table 1 are averages of duplicate tests. These results show such frequent lack of agreement in duplicate tests as to indicate that laboratory tests on plaster panels are of doubtful value if not practically worthless for comparative tests. The tests do clearly show that none of the primers tested can be relied upon to give a satisfactory protection to a second coat from great amounts of water coming through the plaster in such a panel test. In this connection particular attention is called to paint $O$ (aluminum paint), which is the most waterproof paint when used in several coats. This shows less color failure, but 
much worse blistering than any other paint tested. With the exception of No. 20 it will be noted that after the panel was dry (see column 23) all the other coatings were apparently in satisfactory condition for repainting (the blisters had disappeared). The aluminum paint is one of the most waterproof and most impervious paints and if great waterproofness and impermeability were desirable characteristics, this paint (except for its color) should be among the best. The fact that it became permanently blistered, which left it in a bad condition for repainting, indicates that very waterproof paint is particularly dangerous for use on plaster that is not dry. The effect of primers that allowed both blistering and marked color change on wet plaster was decidedly different. Although the color change and accompanying change in gloss were permanent, the blisters that appeared on the wet panels disappeared on thorough drying. The dry panels were apparently in good condition for repainting.

The penetration test, column 10, shows that while this test serves to distinguish between a penetrating and nonpenetrating primer, inspection of the other tests gives no evidence on which one would be justified in giving any preference to either type. Newspaper is recommended over kraft and blotting paper for this test

The tests to indicate ability to prevent spotting (stop "suction") or change of gloss of a second coat (columns 18 to 20, inclusive) are very informative. The test on blotting paper (column 18) appears to be the best of this group, although the painted surfaces on kraft paper (not recorded) were smoother. A paint that fails in this test. should not be considered satisfactory for priming plaster if only one or two additional coats are expected to give a satisfactory appearance. It will be noted that no paint that dried flat on tin (column 14) passed the test for ability to prevent spotting or loss of gloss on both blotting paper, concrete, and plaster. This does not prove that it is not possible to make a satisfactory priming coat paint that dries flat on tin or glass. Neither is it necessary that the paint dry to a noticeable gloss on plaster. (See column 16.) A number of the satisfactory paints dried to not more than an eggshell gloss when applied on plaster. However, those primers that dried flat on tin (No. 9 paint excepted) showed some spotting when applied on plaster and followed by a finishing coat (column 20). It is worth pointing out that of all the commercial plaster primers examined, only six dried flat on tin. It will also be noted (see paint $K$ ) that raw linseed oil with drier and thinner is not a suitable vehicle for a plaster priming coat. This is an example of a paint which dries to a semiglossy surface on tin, but is a poor plaster primer. This paint is too easily affected by the "suction" of porous surfaces, resulting in poor sealing properties.

The tests for brittleness, columns 12 and 13, inclusive, show that a few primers are brittle. While these tests do not give any positive indication of the durability of the primers, it is obvious that a brittle primer should be looked upon with suspicion. These are believed to be rather mild tests for brittleness. For example, paint Q (containing considerable varnish) showed no cracking when brushed on tin and exposed to the carbon arc light for 19 hours. Another panel showed no cracking after being baked for 19 hours at $105^{\circ} \mathrm{C}$. 


\section{RECOMMENDATIONS}

\section{CONDITION OF PLASTER}

The important question as to how dry plaster should be before being painted was not studied in this investigation. The results obtained when the painted panels were imbedded in wet sand show that water may cause color failure, blisters, or loss of gloss. However, the conditions in this test were probably much more severe than those usually encountered in practice, even when painting recently plastered surfaces, except by accidental wetting of the plaster or when ventilation during final drying of the plaster is not sufficient to prevent actual condensation of water on the paint. It is worthy of note that the painted panels used on the "humidity cabinet" showed no marked defects after a month's exposure, even though the inside surfaces of the panels were constantly wet. In these experiments the air in contact with the painted surfaces of the panels was dry enough so that no condensation occurred, and it is not improbable that no liquid water reached the painted surface.

\section{GENERAL CHARACTERISTICS OF A GOOD PLASTER PRIMER}

One coat of good primer for plaster should so efficiently seal a dry porous surface that a single finishing coat of an oil or varnish paint applied on this primed surface will dry with substantially the same appearance that it would have if applied to an impervious material. It'should be of a color satisfactory to the buyer and should have reasonably good hiding power. The dried film should not be brittle, and should preferably dry to at least an eggshell gloss on tin (based upon the data in this paper). There is no evidence to justify preference for either the so-called penetrating or nonpenetrating types of primers. However, primers which contain no binding material in the vehicle except raw, or even boiled linseed oil, penetrate plaster irreguarly and to an undesirable extent. Better sealing and a more uniform finish result from the use of suitable bodied oils or varnish which penetrate less deeply but more uniformly. Finally, the primer should spread easily under the brush and should have good keeping qualities.

\section{HOW TO GET A GOOD PLASTER PRIMER}

It is believed that a specification for a primer can better be based almost entirely on physical or performance tests rather than on requirements of composition. Such a specification has been recommended to several large users and with some changes is given in the appendix. Trial of this specification should in a few years indicate whether it should be made a standard specification. It should be distinctly understood that this specification applies only to the paint used in priming plaster, and not to the finishing coats. While the "comparison primer" is a good primer, in fact one of the best primers made from white lead, it is simply a standard for comparison in the specification. Paints $\mathrm{A}$ and $\mathrm{B}$ of the specification are to be regarded as "reagents" for testing. The "oil gloss finish coat" is an outside white lead paint which would turn yellow on 
interior surfaces, and better formulas can be given for flat white lead paint than the "flat finish coat." The description of the blotting paper used in the test of sealing properties is simply the Government Printing Office requirement for white blotting paper. Any clean, white blotting paper of good quality will probably be equally satisfactory.

For obvious reasons no statement regarding conformity or nonconformity with this specification is given for the individual commercial priming paints recorded in Table 1, though it is apparent that the table indicates that more than one-third of them would pass the specification. The following comments on the experimental (lettered) paints are given:

Paint $K$ does not pass the requirements for sealing properties. In practice an excessive number of coats is frequently necessary, when using this primer.

Paint L meets the specification, although it is not an especially good sealer on porous material like concrete.

On porous surfaces, such as concrete, paint $M$ does not have sufficient sealing properties. It is inferior to paint Q (another white lead paint) in its ability to stop "suction."

Paint $O$ is not white and as noted in Section $V$ is particularly liable to blistering on wet plaster.

Paint $\mathrm{P}$ is brittle, dries flat, and does not pass the test for sealing properties.

Paint Q is a good example of a "penetrating" primer. It is the "comparison primer" of the suggested specification.

Paint $R$ meets the specification. It is a good example of a "nonpenetrating" primer.

\section{ACKNOWLEDGMENT}

The writers are indebted to Clara B. Sentel for most of the rather difficult chemical analyses and also for help in making the various physical tests.

\section{APPENDIX - SUGGESTED SPECIFICATION FOR PAINT FOR PRIMING PLASTER SURFACES (PLASTER PRIMER AND SEALER)}

\section{A. Note.}

This is not a Federal specification, but is suggested for trial by any large user of paint desiring a priming coat for previously unpainted plaster to be finished with two or three coats of oil or varnish paint.

\section{B. Grades and Types.}

$B-1$. - This specification covers one grade of ready-mixed white paint of the bodied oil or varnish type for interior use as a primer on unpainted, old or new, plaster surfaces. It is especially intended to seal and stop the varying "suction" of porous plaster by one coat, and thus prevent absorption of the second coat, and permit a finished job in two or three coats at the most. 


\section{Detail Requirements.}

C-1. Pigment.-The manufacturer is given wide latitude in the selection of the pigments, provided the finished paint passes the requirement for hiding power. This means that some opaque white pigments must be used.

C-2. Liquid.-The liquid shall be any suitable mixture of processed drying oils or varnish with drier, turpentine, volatile mineral spirits or a mixture thereof. The liquid shall contain not less than 42 per cent of nonvolatile matter.

$C-3$. Paint.-The paint, as received, shall be well ground, shall not cake, "liver," thicken, "skin," or settle badly in the container. It shall be readily stirred with a paddle to a smooth paint of suitable brushing consistency for use as a priming coat for unpainted plaster. It shall keep well in the can, after repeated use.

$C-4$. Color.-The paint unless otherwise specified shall be of a good white color. By mutual agreement between buyer and seller, the color shall match that of a sample mutually agreed upon.

$C-5$. Brushing properties. - The paint shall be of a suitable brushing consistency, as it comes from the can, for direct application on new or old unpainted plaster, except where specific manufacturer's directions call for a small amount of additional thinner (turpentine, mineral spirits, or a mixture thereof). Pastes, semipaste paints, or thick-bodied paints are not contemplated under this specification. The paint shall not pull nor have a quick set under the brush. It shall brush as easily, flow and level as readily, and present as uniform a coat as the "comparison primer" described at the end of the specification.

C-6. Penetration on ordinary newspaper.-This specification does not limit a primer to be of either the so-called penetrating or nonpenetrating type. However, the purchaser may specify one of these two types if he so desires. If the nonpenetrating type is specified, this shall be so stated in the invitation for bids, and the test described under paragraph $D-2-e$ shall be included. Otherwise this test shall not be made.

C-7. Hiding power. - The paint direct from the can shall show a wet hiding power by the brushout (checkerboard) method of not less than 110 square feet per gallon (see $D-P-f$ ). If the manufacturer recommends the addition of a definite small amount of thinner to his paint before using, the hiding-power requirement shall apply to the thinned paint.

C-8. Flexibility and adhesion.-The paint as it comes from the can, when normally brushed on tin panels (one coat), allowed to dry for 18 hours, baked for 4 hours at $105^{\circ}$ to $110^{\circ} \mathrm{C}$., and bent over a $3 \mathrm{~mm}$ rod at $24^{\circ} \mathrm{C}$., shall show no evidence of flaking or cracking when viewed at 7 diameters magnification. (See $D-2-g$.)

C-9. Gloss.-The paint when brushed on bright tin panels shall dry with approximately as high a gloss as the "comparison primer." $C-10$. Set to touch (on tin). - The paint shall set to touch on tin in not less than 60 minutes and not more than 4 hours.

C-11. Dry hard (suitable for recoating).-The paint shall dry hard on tin in not more than 18 hours.

C-12. Sealing properties and ability to prevent spotting of the second coat.-The paint as used shall thoroughly seal in one coat 
so as to prevent "suction" spots on succeeding coats, and permit a satisfactory two or three coat job. In these respects, it shall be at least equal in its sealing properties and ability to stop suction spots, to the "comparison primer" used in the test.

D. Methods of Sampling and Testing.

Deliveries will, in general, be sampled and tested by the following methods, but the purchaser reserves the right to use any additional available information to ascertain whether the material meets the specification.

D-1. Sampling.-It is mutually agreed by buyer and seller that not less than one package out of each 1,000 packages shall be taken as representative of each lot of paint. Whenever possible an original unopened package shall be sent to the laboratory, and when this is for any reason not done the inspector shall determine by thorough testing with a paddle or spatula whether the material meets the requirement regarding caking in the container. He shall then thoroughly mix the contents of the container and draw a sample of not less than 1 quart. This sample shall be placed in a clean, dry, metal or glass container, which it must nearly fill. The container shall be closed with a tight cover, sealed, marked, and sent to the laboratory for test with the inspector's report on caking.

When requested, a duplicate sample may be taken from the same package and delivered to the seller, and the inspector may take a third sample to hold for test in case of disagreement.

\section{D-2. Laboratory examination.-}

D-2-a. Caking in container.-When an original package is received in the laboratory it shall be weighed, opened, and stirred with a stiff spatula or paddle. The paint shall be no more difficult to mix to a uniform consistency than any good grade of ready-mixed paint.

D-2-b. Net weight of contents and weight per gallon.-The paint shall be thoroughly mixed, removed from the container, and the container wiped clean and weighed. This weight subtracted from the weight of the original package gives the net weight of the contents. A portion of the thoroughly mixed paint shall be placed in a clean container and portions for the remaining tests promptly weighed out. Weigh a clean, dry, $100 \mathrm{ml}$ graduauted flask. Fill to the mark with the thoroughly mixed paint and weigh again. The increase in weight expressed in grams, divided by 100 , gives the specific gravity, which multiplied by 8.33 gives the weight in pounds per gallon.

If one has a number of such determinations to make, it is convenient to have made a short cylinder of brass about $76 \mathrm{~mm}$ high by $38 \mathrm{~mm}$ inside diameter, with the inside of the bottom rounded, and having a capacity of $83.3 \mathrm{~g} \pm 0.1 \mathrm{~g}$ of water at $20^{\circ} \mathrm{C}$. This cylinder is provided with a counterpoise weight. The paint is poured into the cylinder until it is completely full, the top leveled off with a spatula, and the full cylinder weighed to $\pm 0.5 \mathrm{~g}$. The weight in grams of the contents divided by 10 is the weight per gallon in pounds, and the weight per gallon in pounds multiplied by 0.12 is the specific gravity. 
D-2-C. Color.-Paint the sample and the standard on clean tin or glass, so that the edges touch one another. Let dry 24 hours and compare colors.

D-2-d. Brushing properties, time of dming.-Brush the wellmixed paint on a suitable panel, which may be ground glass, metal, or well-filled wood. Note whether the paint works satisfactorily under the brush. Place the panel in a vertical position in a wellventilated room and let stand for 60 minutes. Note whether the paint has set to touch. Then let the panel stand for 3 hours longer. Note whether the paint has set to touch. Then let the panel stand for 14 hours longer. The paint should be dry, free from streaks, and show a smooth, hard, level finish. Flow a portion of the paint on a clean glass plate and dry in a nearly vertical position at room temperature $\left(70^{\circ}\right.$ to $100^{\circ} \mathrm{F}$.). Note whether the paint streaks or separates within a distance of 4 inches from the top of the film. Brush the paint, likewise, on unpainted plaster panels (new or old) and on at least 4 square feet of white blotting paper. Do the same with the "comparison primer." Note any tendency for the sample under test to pull under the brush or give a quick set on the porous surfaces, as compared with the "comparison primer."

D-Q-e. Penetration on newspaper.-This test is omitted unless specifically called for. (See C-6.) Brush a normal coat of the paint on a sheet of ordinary newspaper. After drying for one hour, examine the reverse side of the paper. There shall be little or no staining through the paper. The painted side shall dry uniformly with a gloss (not flat) within 18 hours.

$D-\mathbb{Z}-f$. Hiding power.-The wet hiding power shall be determined by the brush-out method on an impervious black and white checkerboard surface, total area being 4 square feet, the black and white squares being 4 to 6 inches on the side. The white shall have a brightness of not less than 75 per cent and the black of not more than 8 per cent. Apply the wet paint uniformly, using a 2-inch brush until " complete" hiding (no contrast in brightness) is visible to the eye when the board is viewed normally in north light near a window. The sample (can, brush, and paint) is weighed before and after applying the paint, and the hiding power per unit computed from the amount of paint used. Express in square feet per gallon thus :

Let $A=$ area of black and white surface in square feet.

$S=$ specific gravity of paint.

$W=$ weight in pounds per U. S. gallon.

$G=$ grams of paint applied to $A$.

Then

$H=$ hiding power in square feet per U. S. gallon.

$$
H=\frac{S \times 3,785 \times A}{G} \text { or } H=\frac{W \times 454 \times A}{G}
$$

D-2-g. Flexibitity and adhesion.-Cut a panel from bright tin plate weighing not more than $25 \mathrm{~g}$ nor less than $19 \mathrm{~g}$ per square decimeter $(0.51$ to 0.39 pound per square foot). The panel shall be about 7.5 by $26 \mathrm{~cm}$ (3 by 10 inches) and must be thoroughly cleaned with benzol immediately before using. Place the panel on a whirl- 
ing disk with the center of the panel in line with the center of revolution. Add 20 or $30 \mathrm{ml}$ of the paint previously strained through a No. 200 sieve; whirl for three minutes at 300 revolutions per minute. Cut the panel in half (resulting in two 3 by 5 inch panels). Let stand in a horizontal position at room temperature (not below $21^{\circ}$ C.) for 18 hours. Next, place the panels in a horizontal position in a properly ventilated oven and bake for four hours at $105^{\circ}$ to $110^{\circ}$ C. Remove the panels from the oven and allow to cool at $24^{\circ} \mathrm{C}$. $\left(75^{\circ} \mathrm{F}\right.$.) for one-half hour.

Place the panels with the painted side uppermost over a $3 \mathrm{~mm}$ (one-eighth inch) rod, held firmly by suitable supports, at a point equally distant from the top and bottom edges of the panels, and bend the panels double rapidly. The paint must show no cracking whatsoever at the point of bending when viewed in strong light at a 7 to 10 diameter magnification. For accurate results the bending of the panels should always be done at $24^{\circ} \mathrm{C}$. $\left(75^{\circ} \mathrm{F}\right.$.), for a lowering of the temperature will make the paint more brittle, while an increase in the temperature will make the paint less brittle. The two panels give a check test. If preferred, the paint may be brushed (normal coat) on the tin panels instead of being applied mechanically.

$D-2-h$. Gloss. - Brush the paint on the tin panels just described $(D-2-g)$ and let dry for 24 hours. The paint should dry approximately as glossy as the "comparison primer."

D-Q-i. Sealing properties and ability to prevent suction spots.This very important test should be made in comparison with the "comparison primer." It should preferably be made on new and old, porous (unpainted and unsized) plaster walls or panels. Since it does not appear possible, as a method of test in the laboratory, to describe how to prepare uniform or standard plaster panels, the test is made on white, porous, blotting paper. This paper should be equivalent to the U. S. Government Printing Office standard sample for 1931 , lot No. 356, property No. 1214, calling for the following specifications :

White blotting paper

Bursting strength

Weight 19 by 24,1000

Absorption (1 $\mathrm{ml}$ standard

ink) - Not to exceed 40 seconds.

The blotting paper is cut in panels at least 12 by 12 inches. Brush one liberal coat of the sample and the "comparison primer" on separate sheets of the white blotting paper. Let dry for 24 hours. Then brush over one half of each primed surface a finish coat of oil gloss "paint A," and over the other half of the primed surface a finish coat of flat oil "paint B." Let dry for 24 hours. The resulting finishes shall be as uniform and as glossy and flat, respectively, over the surface primed with the sample under test as over the surface primed with the "comparison primer."

D-Q-j. Comparison paints.-The numbers refer to Federal specification numbers.

\section{"Comparison primer"}

100 pounds stiff paste white lead in oil. TT-W-251, type B.

4 gallons interior varnish. TT-V-71.

2 gallons kettle-boiled linseed oil. JJJ-O-331, type A.

1 gallon turpentine. LLL-T-791. 
"Paint A" (oil gloss finish coat)

100 pounds stiff paste white lead in oil. TT-W-251, type $B$.

3 gallons raw linseed oil. JJJ-O-336.

1 pint turpentine. LLL-T-791.

1 pint drier. TT-D-651.

\section{"Paint B" (flat fnish coat)}

100 pounds stiff paste white lead in oil. TT-W-251, type B.

3 gallons turpentine. LLL-T-791.

$1 / 2$ pint drier. TT-D-651.

Washington, April 12, 1932. 

